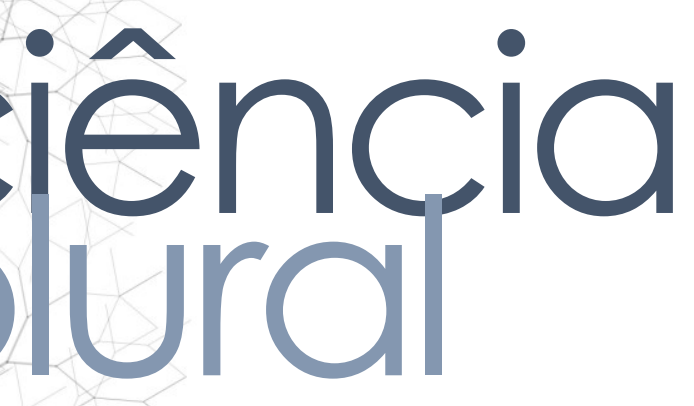

\title{
PERFIL EPIDEMIOLÓGICO DE ACIDENTES COM ANIMAIS PEÇONHENTOS NO ESTADO DO MARANHÃO
}

Epidemiological profile of accidents involving poisony animals in the state of Maranhão

Perfil epidemiológico de accidentes con animales venenosos en el estado del Maranhão

Eduardo Costa Cordeiro $\bullet$ Graduando em Medicina na Universidade Federal do Delta do Parnaíba-UFDPar • E-mail: duduwow20010@hotmail.com

Joelson dos Santos Almeida • Enfermeiro • Mestre em Saúde e Ambiente pela UFMA • Docente do Programa de Residência Multiprofissional em Oncologia da Escola de Saúde Pública do Maranhão-ESP/MA • E-mail: joelsonalmeida2011@gmail.com

Thiago Sousa da Silva • Médico veterinário • Especialista em Saúde Pública • Universidade Federal do Piauí-UFPI • E-mail: thiago17ss@hotmail.com

Autor responsável pela correspondência:

Joelson dos Santos Almeida • E-mail: duduwow20010@hotmail.com 


\section{RESUMO}

Introdução: Acidentes causados por animais peçonhentos são considerados um problema de saúde pública, sendo responsáveis por considerável número de agravos à população mundial. Estudos acerca do tema são escassos no Maranhão, havendo a necessidade de investigação dos casos, visto que são de fundamental importância para as ações de vigilância epidemiológica e ambiental. Objetivo: Descrever o perfil epidemiológico dos acidentes com animais peçonhentos no Estado do Maranhão, Brasil. Metodologia: Trata-se de estudo ecológico, descritivo com abordagem quantitativa. Os dados foram coletados no Sistema de Informação de Agravos de Notificação/Departamento de Informática do Sistema Único de Saúde ocorridos no período de 01 janeiro de 2009 a 31 de dezembro 2019 dos casos notificados de acidentes com animais peçonhentos. Resultados: Constou-se maior incidência de notificações de acidentes em 2019, nos meses de Fevereiro (10,14\%), Março (9,96\%) e Maio (9,89\%). No que tange ao perfil das vítimas houve uma predominância no sexo masculino (68,16\%), adultos jovens de 20 a 39 anos (36,24\%) e autodeclarados pretos (75,03\%). No que diz respeito à gestação a imensa maioria não se aplica a categoria gestante $(77,71 \%)$. Em relação as características do acidente destacou-se o acidente escorpiônico (55,97\%), seguido pelos acidentes com aranhas (30,66\%) e ofídicos (4,09\%). O tempo decorrido entre o acidente e o atendimento foi predominante no intervalo de 1 a 3 horas após o acidente (34,37\%). Dos acidentes ocorridos $61,31 \%$ foram classificados como leves, $82,02 \%$ evoluíram clinicamente com cura e $0,6 \%$ para óbito. Conclusões: Os acidentes causados por animais peçonhentos são de relevância para que haja um planejamento com medidas intersetoriais de vigilância epidemiológica junto a outros órgãos ambientais para minimizar o número de mortes.

Palavras-Chave: Animais Venenosos; Vigilância em Saúde Pública; Mordeduras e Picadas.

\section{ABSTRACT}

Introduction: Acidents caused by venomous animals are considered a public health problem, being responsible for a considerable number of injuries to the world population. Studies on the theme are scarce, with the need to investigate the cases, since they are of fundamental importance for the actions of epidemiological and environmental surveillance. Objective: To describe the epidemiological profile of accidents with venomous animals in the state of Maranhão, Brazil. Methodology: This is an ecological, descriptive study with a quantitative approach. Data were collected at Information System for Notifiable Diseases / Informatics Department of the Unified Health System from January 1, 2009 to December 31, 2019 of the reported cases of accidents with venomous animals. Results: There was a higher incidence of accident notifications in 2019 and in the months of February (10.14\%), March (9.96\%) and May $9.89 \%)$. Regarding the profile of the victims, there was a predominance of males .16\%), young adults aged 20 to 39 years (36.24\%) and self-declared blacks $(75.03 \%)$. ith regard to pregnancy, the vast majority does not apply to the pregnant category $71 \%)$. Regarding the characteristics of the accident, the scorpionic accident stood $(55.97 \%)$, followed by accidents with spiders (30.66\%) and snakebites $(4.09 \%)$. The elapsed between the accident and the assistance was predominant in the interval 
from 1 to 3 hours after the accident (34.37\%). Of the accidents that occurred, $61.31 \%$ were classified as mild, $82.02 \%$ progressed clinically with cure and $0.6 \%$ died. Conclusions: Accidents caused by venomous animals are relevant for planning intersectoral measures for epidemiological surveillance with other environmental agencies to minimize the number of deaths.

Keywords: Poisonous Animals; Public Health Surveillance; Bites and Stings.

\section{RESUMEN}

Introducción: Los accidentes ocasionados por animales venenosos son considerados un problema de salud pública, siendo responsable de un número considerable de lesiones a la población mundial. Los estudios sobre el tema son escasos, con la necesidad de investigar los casos, ya que son de fundamental importancia para las acciones de vigilancia epidemiológica y ambiental. Objetivo: Describir el perfil epidemiológico de accidentes con animales venenosos en el estado de Maranhão, Brasil. Metodología: Se trata de un estudio ecológico, descriptivo con enfoque cuantitativo. Los datos fueron recolectados en el Sistema de Información de Enfermedades Notificables / Departamento de Informática del Sistema Único de Salud que ocurrieron en el período del 01 de enero de 2009 al 31 de diciembre de 2019 de los casos notificados de accidentes con animales venenosos. Resultados: Hubo una mayor incidencia de notificaciones de accidentes en 2019 y en los meses de febrero (10,14\%), marzo $(9,96 \%)$ y mayo $(9,89 \%)$. En cuanto al perfil de las víctimas, predominó el sexo masculino (68,16\%), adultos jóvenes de 20 a 39 años $(36,24 \%)$ y negros autodeclarados $(75,03 \%)$. En cuanto al embarazo, la gran mayoría no aplica a la categoría de embarazadas $(77,71 \%)$. En cuanto a las características del accidente, destacó el accidente escorpiónico (55,97\%), seguido de los accidentes con arañas (30,66\%) y las mordeduras de serpientes $(4,09 \%)$. El tiempo transcurrido entre el accidente y la asistencia fue predominante en el intervalo de 1 a 3 horas posteriores al accidente (34,37\%). De los accidentes ocurridos, $61,31 \%$ fueron clasificados como leves, $82,02 \%$ progresó clínicamente con curación y 0,6\% fallecieron. Conclusiones: Los accidentes causados por animales venenosos son relevantes para planificar medidas intersectoriales de vigilancia epidemiológica con otras agencias ambientales para minimizar el número de muertes.

Palabras clave: Animales venenosos; Vigilancia en Salud Pública; Mordeduras y picaduras. 


\section{Introdução}

Os acidentes causados por animais peçonhentos são considerados como um problema de saúde pública relevante, contudo somente em 2009 a Organização Mundial da Saúde (OMS) passou a considerar os acidentes causados por animais peçonhentos como integrantes da lista das principais Doenças Tropicais Negligenciadas no mundo ${ }^{1}$.

No continente americano, especificamente no Brasil, os escorpiões, ofídios e aranhas fazem parte de um extenso grupo de animais capazes de provocar envenenamentos em seres humanos, sendo estes importantes agentes epidemiológicos que cursam com alterações de gravidade leve a severa, podendo ser responsabilizados por cerca dos mais de 100 mil acidentes e pelos quase 200 óbitos catalogados por ano, além disso, outros animais peçonhentos ou venenosos podem representar percentual $(5,8 \%)$ significativo desses casos e juntos constituem a segunda maior causa de intoxicações humanas no país ${ }^{2,3}$.

Quanto aos fatores condicionantes tem-se notado que a expansão demográfica e as alterações antrópicas provocadas no habitat natural desses espécimes interferem de forma significativa em suas cadeias alimentares e em seus abrigos, o que resulta por sua vez, na migração à espaços urbanos ou rurais como por exemplo residências, construções e terrenos baldios ${ }^{4}$.

Aponta-se também que alterações climáticas, como o aumento dos índices pluviométricos, baixo nível de escolaridade, condições de trabalho insalubres e precárias dentre outras, são aspectos favoráveis ao aumento do número de casos desses acidentes, tendo em vista que os acidentes de trabalho (AT) ocorridos com indivíduos ocupados com atividades relacionadas a ambientes de trabalho rural, florestas e construção civil lideram os números notificados além dos relatados em crianças e donas de casa $2,5,6$.

Na região avaliada estudos epidemiológicos acerca do tema são inexistentes, sim a realização de tal pesquisa é fundamental para que tenhamos um mapeamento quadro de morbimortalidade, bem como, informações consistentes para a stituição de um banco de dados referentes a estes acidentes ocorridos no Estado. 
Partindo desta problemática, o estudo objetiva descrever o perfil epidemiológico dos acidentes com animais peçonhentos no estado do Maranhão, Brasil.

\section{Metodologia}

Trata-se de um estudo ecológico, descritivo com abordagem quantitativa. A pesquisa foi realizada no estado do Maranhão no período de março a abril de 2020, na qual foram analisados os casos registrados no Sistema de Informação de Agravos de Notificação vinculado ao Departamento de Informática do Sistema Único de Saúde (SINAN/DATASUS).

A população do estudo foi composta dos casos notificados por acidentes com animais peçonhentos, apenas da população residente no estado do Maranhão. Dessa forma, a amostra foi constituída pelas notificações do Sistema de Informação de Agravos de Notificação vinculado ao Departamento de Informática do Sistema Único de Saúde (SINAN/DATASUS) ocorridas no período de 01 janeiro de 2009 a 31 de dezembro 2019 no estado do Maranhão.

O critério de inclusão se constitui: casos notificados de ambos os sexos que tenha sido acidentado no período do estudo, ser componente da amostra da população residente, possuir registro da ficha de notificação para acidentes com animais peçonhentos no Sistema de Informação de Agravos de Notificação vinculado ao Departamento de Informática do Sistema Único de Saúde (SINAN/DATASUS) com dados do estado do Maranhão. Foram desconsiderados os casos fora do período de estudo, casos notificados da população não residente no estado do Maranhão e casos não concluídos.

A coleta de dados ocorreu através de dados disponibilizados do SINAN/DATASUS que estão publicados na página do DATASUS (http://tabnet.datasus.gov.br/cgi/deftohtm.exe?sinannet/cnv/animaisma.def) em modo público sendo extraído em uma planilha do Excel, na qual constam os dados rupados dos casos notificados de acidentes com animais peçonhentos no período do udo. O instrumento de coleta foi baseado na ficha de notificação/investigação de dente com animais peçonhentos na qual foram analisadas as variáveis deste estudo 
sendo as seguintes: sexo, faixa etária, cor, escolaridade, presença de gestação, causador do acidente, causado por serpente, causado por aranha, tempo entre picada e atendimento, classificação do acidente e evolução do caso.

Os dados disponíveis no SINAN/DATASUS foram exportados e tabulados utilizando o programa Microsoft Office Excel versão 2019, os dados populacionais necessários para o cálculo das taxas incidência, letalidade foram obtidos a partir do Instituto Brasileiro de Geografia e Estatística (IBGE), sendo que no ano de 2010 serão considerados os dados do censo e nos demais anos $(2009,2011,2012,2013,2014,2015$, 2016, 2017, 2018, 2019) as projeções intercensitárias, disponível no endereço eletrônico: http://tabnet.datasus.gov.br/cgi/deftohtm.exe?novapop/cnv/popbr.def e https://www.ibge.gov.br/apps/populacao/projecao/.

A análise dos dados ocorreu por meio estatística descritiva na qual foram organizados os dados de notificação sendo utilizadas as frequências absolutas e relativas nas variáveis do estudo, posteriormente processados no software Stata 14.0, sendo expressos por meio de figuras e tabelas.

Esta pesquisa foi desenvolvida em conformidade a resolução $n^{\circ} 466$ de 12 de dezembro de 2012 do Conselho Nacional de Saúde e da resolução no 510 de 7 de abril de 2016, que trata sobre as diretrizes e normas de pesquisa envolvendo seres humanos.

Por se tratar de uma pesquisa com dados secundários disponibilizados em modo público no site do DATASUS/SINAN vinculado ao Ministério da Saúde não foi necessário à avaliação para apreciação e aprovação do comitê de ética em Pesquisa.

\section{Resultados}

No período de 2009 a 2019 foram notificados 30.702 casos de acidentes por animais peçonhentos no estado do Maranhão. A partir dos dados obtidos, as taxas com maiores incidências foram nos anos de 2019 ( $n=5.058 ;$ 71,49/100000hab), 2018 $n=4.622 ; 65,7 / 100000 \mathrm{hab})$ e $2017(\mathrm{n}=3.458 ; 49,4 / 100000 \mathrm{hab})$ delineando uma curva crescente. E a menor incidência foi em 2009 (n=1801; 28,29/100000hab) (Figura 1). 


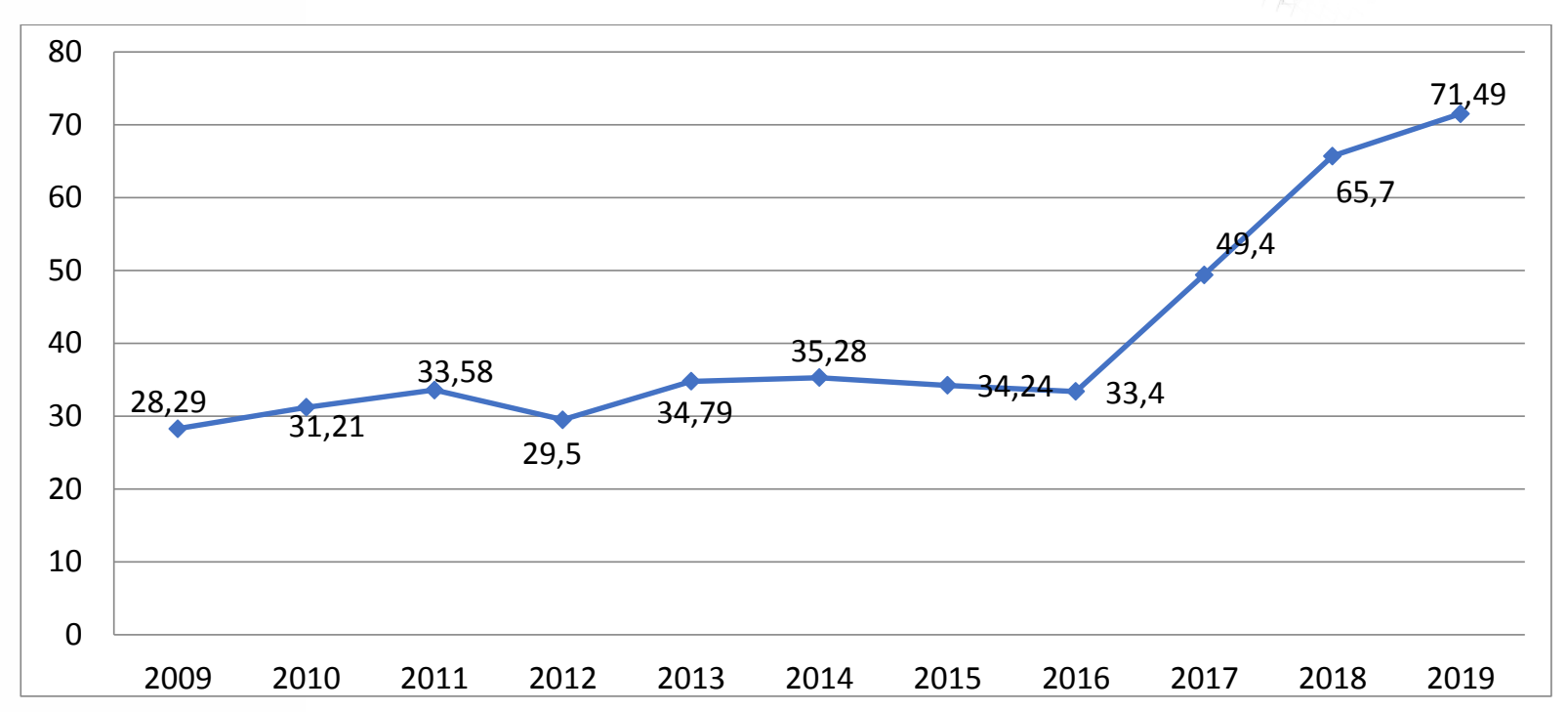

Figura 1: Taxa de incidência dos acidentes por animais peçonhentos por ano de ocorrência no período de 2009 a 2019, n=30.702, no estado do Maranhão, Brasil, São Luís - MA, 2020, Sistema de Informação de Agravos de Notificação.

Em relação aos meses, a maior ocorrência de acidentes com animais peçonhentos, na série histórica analisada, ocorreu nos meses de Janeiro $(n=3112$; $10,14 \%)$, Fevereiro ( $n=3.058 ; 9,96 \%)$, Março $(n=2.992 ; 9,75 \%)$ e Maio $(n=3.037 ; 9,89 \%)$. Isso se deve aos altos índices pluviais durante os primeiros meses do ano e ao clima quente da região que favorece a proliferação dos artrópodes (Figura 2).

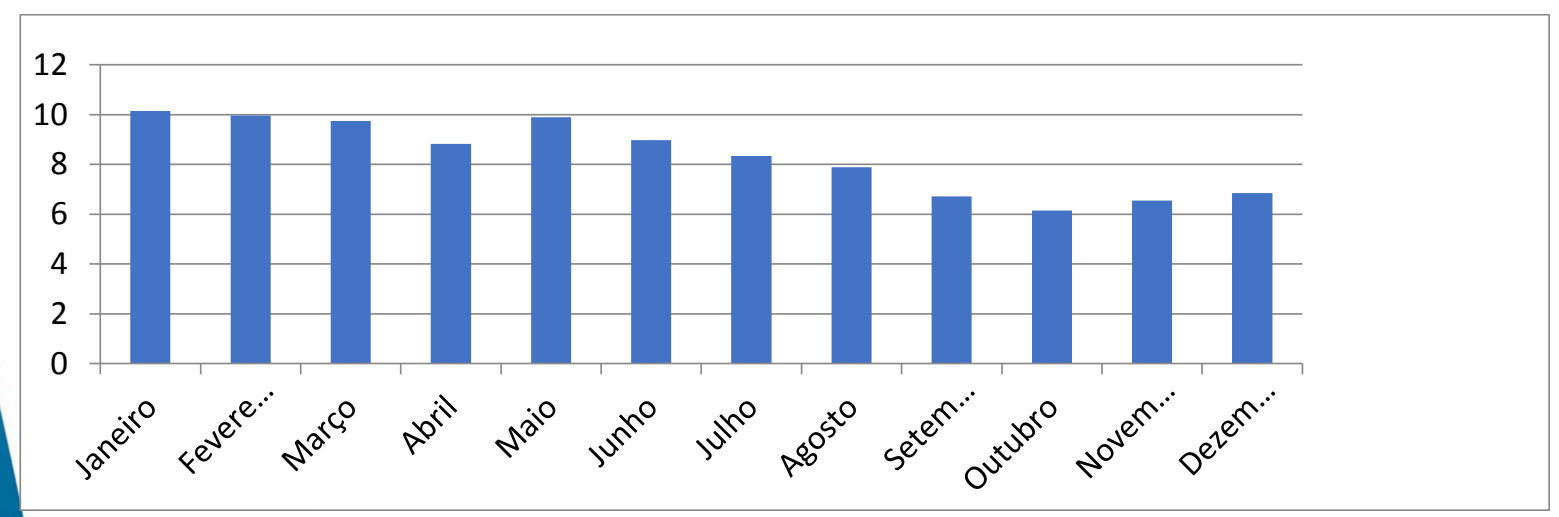

Figura 2: Distribuição dos meses de ocorrência dos acidentes por animais peçonhentos no Estado do Maranhão, (2009-2019), n=30.702, Brasil, São Luís - MA, 2020, Sistema de Informação de Agravos de Notificação.

Com relação ao perfil sociodemográfico mostraram que $68,16 \%$ (n=20.925), era exo masculino, com faixa etária entre 20 a 29 anos cerca de 36,24\% (n=11.126) e a plaridade foi ignorada na maioria dos casos 93,12\% ( $\mathrm{n}=28.590)$. Das vítimas por entes com animais peçonhentos, a maioria se autodeclarou preta 75,03\% 
$(n=23.036)$. No quesito gestante, $77,61 \%$ (23.828) não se aplica devido à maioria dos casos serem do sexo masculino (Tabela 1).

Tabela 1: Perfil socioeconômico e clínico das vítimas de acidentes por animais peçonhentos notificados no Estado do Maranhão (2009-2019), n=30.702, São Luís - MA, Brasil, 2020, Sistema de Informação de Agravos de Notificação.

\begin{tabular}{|c|c|c|}
\hline VARIÁVEIS & $\mathrm{N}=30.702$ & $\%$ \\
\hline \multicolumn{3}{|l|}{ Sexo } \\
\hline Masculino & 20.925 & 68,16 \\
\hline Feminino & 9.774 & 31,83 \\
\hline Ignorado & 3 & 0,01 \\
\hline Faixa etária (anos) & $\mathbf{N}$ & $\%$ \\
\hline$<1$ & 471 & 1,53 \\
\hline $1-4$ & 1.022 & 3,33 \\
\hline $5-9$ & 1.811 & 5,9 \\
\hline $10-14$ & 2.529 & 8,24 \\
\hline $15-19$ & 2.972 & 9,68 \\
\hline $20-39$ & 11.126 & 36,24 \\
\hline $40-59$ & 7.501 & 24,43 \\
\hline $60-69$ & 2086 & 6,8 \\
\hline $70-79$ & 913 & 2,97 \\
\hline$>79$ & 267 & 0,87 \\
\hline Ignorado/Branco & 4 & 0,01 \\
\hline \multicolumn{3}{|l|}{ Escolaridade } \\
\hline Nenhuma & 2.112 & 6,88 \\
\hline Ignorado/Branco & 28.590 & 93,12 \\
\hline Cor/Raça & $\mathbf{N}$ & $\%$ \\
\hline Amarelo & 2.655 & 8,65 \\
\hline Branco & 2.641 & 8,60 \\
\hline Pardo & 451 & 1,47 \\
\hline Preto & 23.036 & 75,03 \\
\hline Indígena & 1.105 & 3,60 \\
\hline
\end{tabular}




\begin{tabular}{|lll|}
\hline Ignorado/Branco & 815 & 2,65 \\
\hline Gestante & $\mathbf{N}$ & $\%$ \\
\hline 1 trimestre & 48 & 0,16 \\
\hline 2 trimestre & 85 & 0,28 \\
\hline 3 trimestre & 62 & 0,20 \\
\hline Idade gestacional ignorada & 67 & 0,22 \\
\hline Não & 6.078 & 19,80 \\
\hline Não se aplica & 23.828 & 77,61 \\
\hline Ignorado/Branco & 534 & 1,73 \\
\hline
\end{tabular}

Os maiores causadores de acidentes foram os escorpiões 55,97\% ( $n=17.183)$, seguido pelas aranhas $30,66 \%(n=9.415)$ e as serpentes $4,09 \%(n=1.257)$.

Dos acidentes causados por serpentes, o gênero Bothrops, família Viperidae, (jararaca, jararacuçu), apresentou maior prevalência 37,42\% (n=11.490), embora na maioria dos acidentes a identificação da espécie tenha sido ignorada/branco 47,53 \% $(n=14.531)$.

Nos acidentes causados por aranhas em 97,43\% (n=29.912) a identificação foi ignorada/branco, seguido pelas (aranhas marrons) da espécie Loxosceles spp, família Sicariidae, 1,11\% ( $\mathrm{n}=342)$ das notificações (Tabela 2).

Tabela 2: Distribuição dos animais peçonhentos causadores de acidentes no Estado do Maranhão (2009-2019), n=30.702, São Luís-MA, Brasil, 2020, Sistema de Informação de Agravos de Notificação.

\begin{tabular}{|lll|}
\hline VARIÁ VEIS & $\mathrm{N}=30.702$ & $\%$ \\
\hline Causador do acidente & $\mathbf{N}$ & $\%$ \\
\hline Escorpião & 17.183 & 55,97 \\
\hline Serpente & 1.257 & 4,09 \\
\hline Aranha & 9.415 & 30,66 \\
\hline Abelha & 576 & 1,88 \\
\hline Lagarta & 1.025 & 3,34 \\
\hline
\end{tabular}




\begin{tabular}{|lll|}
\hline Outros & 964 & 3,14 \\
\hline Ignorado/Branco & 282 & 0,92 \\
\hline Causado por Serpente & $\mathbf{N}$ & $\%$ \\
\hline Bothrops spp. (jararaca, jararacuçu) & 11.490 & 37,42 \\
\hline Crotalus spp. (cascavel) & 3.991 & 12,99 \\
\hline Micrurus corallinus (Coral- & 134 & 0,44 \\
\hline Lachesis (surucucu, surucucu-de- & 92 & 0,30 \\
\hline Não peçonhenta & 466 & 1,52 \\
\hline Ignorado/Branco & 14.531 & 47,33 \\
\hline Causado por Aranha & $\mathbf{N}$ & $\mathbf{0}$ \\
\hline Phoneutria spp. (aranha-armadeira) & 82 & 0,27 \\
\hline Loxosceles spp. (aranha-marrom) & 342 & 1,11 \\
\hline Latrodectus spp. (viúva-negra) & 13 & 0,04 \\
\hline Outras espécies & 353 & 1,43 \\
\hline Ignorado/Branco & 29.912 & \\
\hline
\end{tabular}

No que tange a classificação final, a maioria dos casos foi leve 61,31\% ( $n=18.823)$ com evolução de cura 82.05\% ( $\mathrm{n}=25.1920)$, apenas $0,60 \%(\mathrm{n}=183)$ dos acidentes evoluíram com óbito. Com relação ao tempo decorrido entre o acidente e o atendimento da vítima é imprescindível para a determinação do seu prognóstico. Em $34,37 \%(n=10.550)$ as vítimas receberam atendimento entre 1 e 3 horas (Tabela 3).

Tabela 3: Distribuição da classificação, tempo decorrido entre a picada/atendimento e evolução dos casos de acidentes causados por animais peçonhentos no período de 2009 2019, n=30.702, no estado do Maranhão, São Luís - MA, Brasil, 2020, Sistema de Informação de Agravos de Notificação.

\begin{tabular}{|lll|}
\hline VARIÁ VEIS & $\mathbf{N}=30.702$ & $\%$ \\
\hline Classificação final & & \\
\hline Ignorado/Branco & 2.716 & 8,85 \\
\hline Leve & 18.823 & 61,31 \\
\hline
\end{tabular}




\begin{tabular}{|lll|}
\hline Moderado & 8.032 & 26,16 \\
\hline Grave & 1.131 & 3,68 \\
\hline Evolução do caso & $\mathbf{N}$ & $\%$ \\
\hline Ignorado/Branco & 5.322 & 17,33 \\
\hline Cura & 25.192 & 82,05 \\
\hline Óbito & 183 & 0,60 \\
\hline Óbito por outras causas & 5 & 0,02 \\
\hline Tempo entre a picada e o atendimento & $\mathbf{N}$ & $\%$ \\
\hline Ignorado/Branco & 2.049 & 6,68 \\
\hline 0-1 & 8.759 & 28,53 \\
\hline 1-3 & 10.550 & 34,37 \\
\hline 3-6 & 4.805 & 15,65 \\
\hline 6-12 & 1.754 & 5,71 \\
\hline 12-24 & 1.384 & 4,50 \\
\hline Maior que 24 & 1.401 & 4,56 \\
\hline
\end{tabular}

\section{Discussão}

O estado do Maranhão apresentou nos anos 2017 (49,4/100.000hab), 2018 (65,7/100.000hab) e 2019 (71, 49/100.000hab) uma maior incidência de notificações de acidentes causados por animais peçonhentos. Os dados encontrados na literatura ${ }^{7}$ são inferiores ao nosso estudo, porém, apresenta incidência com picos nos anos de 2009 (27,25/100.000hab), 2010 (26,75/100.000hab) e 2011 (27,59/100.000hab). Essa divergência pode ser explicada pela ausência ou subnotificações dos acidentes ou por negligência a problemática.

Em relação ao meses Janeiro (10,14\%), Fevereiro (9,96\%) e Maio $(9,89 \%)$ resentam maior sazonalidade e com isso, os acidentes apresentam maior incidência. es dados são semelhantes aos encontrados no estado de Minas Gerais e na cidade Nova Xantina-MT ${ }^{4,8}$. Esse fato pode ser explicado pela prevalência de chuva nos 
meses supracitados e o clima quente da região que ofertam condições ideais para a sobrevivência e reprodução de animais peçonhentos ${ }^{9,10}$.

Os dados revelaram que indivíduos do sexo masculino apresentaram maior prevalência de acidentes $(68,16 \%)$ quando comparado às estatísticas do sexo feminino. Estudos semelhantes corroboram com o achado, evidenciando uma quantidade maior de acidentes em homens ${ }^{11,12}$.

Observou-se que a faixa etária mais acometida foi de jovens adultos (20 a 39 anos), economicamente ativos, com escolaridade ignorada e de cor preta (75,03\%), tais achados condizem com estudos que mostram uma maior incidência de acidentes em homens jovens economicamente ativos e em situação de vulnerabilidade social 4,8,13,14, 15.

Em relação a variável raça/cor, houve uma prevalência de acidentes em pessoas autodeclaradas pretas com 23.036 (75,05\%), seguida de pessoas autodeclaradas amarelas com $2.655(8,65 \%)$ casos. Esse fato pode ser explicado por uma maior proporção de indivíduos autodeclarados pretos no estado do Maranhão, segundo dados do IBGE16.

No que diz respeito às gestantes grande parte dos casos notificados foram caracterizados no quesito "não se aplica". Isso ocorre devido à maioria dos acidentes terem acometidos homens, no caso de mulheres fora do período fértil como, por exemplo, os extremos de idade. Entretanto, dos acidentes ocorridos à maioria se concentrou no segundo trimestre de gravidez, o qual constitui um importante fator de risco devido às ações proteolíticas, coagulantes e/ou hemorrágicas dos venenos, aumentando o índice de hemorragia uterina e, consequentemente, o óbito materno e fetal 17 .

Em relação aos animais peçonhentos, os acidentes causados por escorpiões foram os mais prevalentes corroborando com o estudo realizado no estado do Piauí e cidade de Nova Xantina-MT ${ }^{8}, 13$. A aranha-marrom da espécie Loxoceles amazônica rtencente à família Sicariidae, apresentou maior índice de notificações. Os acidentes vocados por aranhas ocuparam a segunda posição, seguido pelos acidentes icos, sendo a jararaca ou jararacuçu do gênero Bothrops pertencente à família 
Viperidae, que apresentam resultados semelhantes encontrados em outros estudos ${ }^{13}$, 15. Esses achados estão de acordo com as espécies de importância médica encontradas no estado do Maranhão que são o (escorpião-do-nordeste) do gênero Tityus stigmurus pertencente à família Buthidae, a (jararaca-do-norte) do gênero Bothrops atrox pertencente à família Viperidae, a (cascavel) do gênero Crotalus durissus pertencente à família Viperidae, a (cobra-coral) do gênero Micrurus ibiboboca pertencente à família Elapidae, e a (aranha-marrom) do gênero Loxoceles amazônica pertencente à família Sicariidae, segundo dados levantados por Silva e et al ${ }^{4}$. Os dados notificados mostraram um despreparo na identificação do agente causador dos acidentes ofídicos, a despeito de sua importância para a instituição do adequado tratamento e prognóstico do paciente ${ }^{17}$.

Com relação ao tempo decorrido entre o acidente e o atendimento predominou no intervalo de 1 a 3 horas. Tais dados são condizentes com encontrados nos estudos realizados no estado do Rio de Janeiro e Minas Gerais ${ }^{4}, 18$. Ademais, dos acidentes ocorridos aproximadamente $14 \%$ das vítimas obtiveram um atendimento com intervalo maior que 6 horas desde a inoculação do veneno. Dessa forma, o desfecho dos acidentes tende a ser pior quanto maior for o intervalo entre a picada e o início do tratamento.

Dos acidentes ocorridos 18.823 no estado do Maranhão foram classificados como leves (61,31\%), ou seja, as vítimas apresentaram como sintomas dores locais e edema, sem grandes repercussões sistêmicas como hemorragias, insuficiência renal, hipotensão arterial e choque ${ }^{2}$.

No que concerne à evolução dos casos, aproximadamente $82 \%$ dos casos evoluíram para cura e somente 0,6\% tiveram como desfecho o óbito. Resultados semelhantes foram encontrados nos estudos de Benício e Silva ${ }^{4,13}$.

É importante destacar que a maioria dos acidentes por animais peçonhentos no stado do Maranhão não obtiveram a correta identificação do agente causador. Isso ode comprometer as estratégias de enfretamento, a melhoria do atendimento clínico, yolução do caso e o prognóstico da vítima, uma vez que sua identificação auxilia no reto tratamento. 


\section{Conclusões}

Os acidentes causados por animais peçonhentos se configura como um problema de saúde pública na qual é possível inferir que o aumento dos índices pode ser explicado pelas modificações antrópicas na natureza. Isto é, a invasão urbana no habitat natural desses animais provoca maior contato do ser humano com animais venenosos. No estado do Maranhão, os índices aumentaram nos últimos três anos ocorrendo, especialmente, nos quatro primeiros meses do ano. Isso se deve ao clima quente e úmido propicio a proliferação dos artrópodes, os maiores causadores de acidentes, principalmente, os escorpiões.

Diante dos dados contidos nesse estudo, é possível inferir que os jovens adultos do sexo masculino são mais vulneráveis a acidentes causados por animais peçonhentos, apresentam uma sintomatologia leve e evoluem para cura se o atendimento for realizado em tempo hábil.

Os estudos ecológicos apresentam como principal limitação à associação da causa de exposição e o evento da qual não se podem avaliar individualmente as pessoas. Dessa forma, à junção entre a exposição e evento ao conjunto populacional não possibilita determinar quando a exposição é identificada nos sujeitos que desenvolvem a patologia, dita, falácia ecológica. Entretanto, pesquisas dessa natureza auxiliam a verificar quais os fatores deve ser investigados para uma maior competência analítica dos fatos.

Portanto, a adoção de medidas intersetoriais entre a vigilância epidemiológica, a secretaria do meio ambiente e o centro de controle de zoonoses são essenciais para a diminuição da mortalidade por acidentes com animais venenosos, através da capacitação profissional e da educação popular em saúde para a correta identificação do agente causador e adoção de medidas de proteção individual. 


\section{Referências}

1. Gutiérrez JM, Theakston RDG, Warreall DA. Confronting the neglected problem of snake bite envenoming: the need for a global partnership. PLoS Med[periódicos na internet]. 2006 jun [Acesso em 25 abr 2020]; 3(6). Disponível em:https://journals.plos.org/plosmedicine/article?id=10.1371/journal.pmed.003015 0. doi: 10.1371/journal. pmed.0030150.

2. Ministério da saúde. Secretaria de Vigilância em saúde. Departamento de Vigilância epidemiológica. Manual de controle de escorpiões, secretaria de Vigilância em saúde, Departamento de Vigilância epidemiológica. Brasília: Ministério da Saúde; 2009.

3. Ministério da Saúde. Portal Brasil. Ministério da Saúde alerta sobre animais peçonhentos: prevenção de acidentes. Brasília: Ministério da Saúde; 2014 [acesso em 25 de abr 2020]. Disponível em: http://www.brasil.gov.br/saude/2013/11/ministerioda-saude-alerta-sobreanimais-peconhentos-1.

4. Silva PLN, Costa AA, Damasceno RF, Neta AIO, Ferreira IS. Perfil epidemiológico dos acidentes por animais peçonhentos notificados no Estado de Minas Gerais durante o período de 2010-2015. Revista Sustinere 2017; 5(2):199-217.

5. Carmo EA, Nery AA, Jesus SC, Casotti CA. Internações hospitalares por causas externas envolvendo contato com animais em um hospital geral do interior da Bahia, 2009-2011. Epidemiol Serv Saúde [periódicos na internet]. 2016 [ acesso em 25 abr 2020]; 25(1):105-14. Disponível em: https://doi.org/10.5123/s167949742016000100011.

6. Sousa FNF, Santana VS. Mortalidade por acidentes de trabalho entre trabalhadores da agropecuária no Brasil, 2000-2010. Cad Saúde Pública [periódicos na internet]. Jan 2016 [acesso em 25 abr 2020]; 32(4). Disponível em: http:/ / www.scielo.br/scielo.php?script=sci_arttext\&pid=S0102311X2016000400711\& lng=pt\&tlng=pt . doi: 10.1590/0102-311X00071914>.

7. Ministério da Saúde, Secretaria de Vigilância em Saúde. Boletim Epidemiológico. Acidentes de trabalho por animais peçonhentos entre trabalhadores do campo, floresta e águas, Brasil 2007 a 2017. Mar 2019; 50(11):1-14.

Santana VTP, Suchara EA. Epidemiologia dos acidentes com animais eçonhentos registrados em Nova Xavantina-MT. Rev epidemiol control infect 2015; (3):141-146.

Nodari FR, Leite ML, Nascimento E. Aspectos demográficos, espaciais e porais dos acidentes escorpiônicos ocorridos na área de abrangência da $3^{\mathrm{a}}$ Regional 
de Saúde-Ponta Grossa, PR, no período de 2001 a 2004. Publicatio UEPG: Ciências Biológicas e da Saúde 2006; 12(1).

10. Oliveira RSA. Variação estacional e anual da precipitação e da temperatura do ar na cidade de Barreirinhas/MA. Chapadinha. Monografia [Bacharel em Agronomia]- Universidade Federal do Maranhão; 2016.

11. Silva CJ, Jorge MT, Ribeiro LA. Epidemiology of snakebite in a central region of Brazil. Toxicon 2003 Feb; 41(2): 251-255.

12. Moreno E, Andrade MQ, Silva RML, Tavares Neto J. Características clínicoepidemiológicas dos acidentes ofídicos em Rio Branco, Acre. Rev Soc Bras Med Trop 2005 Feb; 38(1): 15-21.

13. Benício RA, Carvalho LS, Fonseca MG. Venomous Animals of State of Piauí: Epidemiology of Accidents and List of Medical Importance Species. Rev Bras Zoo 2019; 20(1):1-14.

14. Costa BC. Fatores de risco para acidentes com escorpiões: uma revisão de literatura. Governador Valadares. Monografia [Especialização em Atenção Básica em Saúde da Família] - Universidade Federal de Minas Gerais, 2011.

15. Leite RS, Targino ITG, Lopes YACF, Barros RM, Vieira AA. Epidemiology of snakebite accidents in the municipalities of the state of Paraíba, Brazil. Cien Saude Colet [periódicos na internet]. 2013 maio [acesso em 25 abr 2020]; 18(5):1463-71. Disponível

em: http:/ / www.scielo.br/scielo.php?script=sci_arttext\&pid=S141381232013000500032\& lng=en\&tlng=en. doi: 10.1590/ S1413-81232013000500032.

16. Instituto Brasileiro de Geografia e Estatística [homepage na internet]. Características da população e dos domicílios: resultados do universo [acesso em abr 2020].

Disponível

em: http:// www.ibge.gov.br/home/estatistica/ populacao/censo20020/caracteristicas_ da_populacao/resultados_do_universo.pdf.

17. Fundação Nacional de Saúde. Manual de Diagnóstico e Tratamento por Animais Peçonhentos. 2. ed. Brasília: FUNASA; 2001.

18. Lima JS, Martelli Júnior H, Martelli DRB, Silva MS, Carvalho SFG, Canela JR, et 1. Perfil dos acidentes ofídicos no norte do Estado de Minas Gerais, Brasil. Rev da Soc Bras Med Trop 2009 Out; 42(5): 561-564.

Submetido em 28/04/2020

Aceito em 15/01/2021 